\title{
MORSE THEORY WITHOUT CRITICAL POINTS
}

\author{
WOLFGANG SMITH
}

Let $X$ denote an $n$-dimensional differentiable manifold and $f: X \rightarrow$ $R$ a real-valued differentiable function on $X$, where "differentiable" means (let us say) $C^{x}$. We shall be concerned with the case where $f$ has no critical points, and thus, too, with the case where $X$ is not compact. In place of critical points we will introduce a notion of "critical fibers", and in place of the index we shall assign to each isolated critical fiber a set of "type numbers" $m_{p}^{+}$for $p=0,1, \ldots, n-1$. Roughly speaking, a critical fiber is one across which the fiber-structure of $f$ suffers a discontinuity, and $m_{p}^{+}$is a homology measure of that discontinuity on dimension $p$. Given that $f$ is bounded and has only a finite number of critical fibers, we let $M_{p}^{+}$denote the sum of the type numbers $m_{p}^{+}$over all critical fibers. Our main result is that these coefficients satisfy the strong Morse inequalities:

$$
\begin{aligned}
M_{0}^{+} & \geq R_{0} \\
M_{1}^{+}-M_{0}^{+} & \geq R_{1}-R_{0} \\
M_{n-1}^{+}-M_{n-2}^{+}+\cdots \pm M_{0}^{+} & =R_{n-1}-R_{n-2}+\cdots \pm R_{0},
\end{aligned}
$$

where $R_{p}$ denotes the p-dimensional Betti number of $X$ (with respect to a given coefficient module $\mathrm{G}$ ). We show, moreover, that for $p<n-1$ they constitute in fact a bona fide generalization of the classical Morse inequalities. For, if $h: M \rightarrow R$ denotes a differentiable function on a compact manifold with non-degenerate critical points, and we let $X$ denote the complement of the critical points in $M$, then our preceding inequalities for $f=h \mid X$ reduce (as will be shown) to the Morse inequalities for $h$ on dimensions $p<n-1$.

1. Basic lemmas. First some notation and terminology. The symbol $H_{p}(X, A)$ will denote the p-dimensional singular homology group of the topological pair $(X, A)$ over some (fixed) coefficient group $G$. We will say that the pair $(X, A)$ is regular if the inclusion $A \subset X$ induces isomorphisms $H_{p}(A) \approx H_{p}(X)$ for all $\mathrm{p}$. We will need the following elementary fact regarding excisive couples [5]:

Copyright $\mathcal{O} 1989$ Rocky Mountain Mathematics Consortium 
LEMMA 1. Let $\left\{X_{1}, X_{2}\right\}$ be an excisive couple such that $\left(X_{2}, X_{1} \cap X_{2}\right)$ is regular. Then $\left(X_{1} \cup X_{2}, X_{1}\right)$ is regular as well.

To show this, one observes that for an excisive couple $\left\{X_{1}, X_{2}\right\}$ one has an inclusion-induced isomorphism (see p. [5, p. 188])

$$
H_{*}\left(X_{2}, X_{1} \cap X_{2}\right) \approx H_{*}\left(X_{1}, \cup X_{2}, X_{1}\right) \text {. }
$$

But since $\left(X_{2}, X_{1} \cap X_{2}\right)$ is regular, it follows that $H_{*}\left(X_{2}, X_{1}, \cap X_{2}\right)$ is identically zero. This implies by (1.1) that $H_{*}\left(X_{1} \cap X_{2}, X_{1}\right)$ is likewise zero, which again implies that the pair $\left(X_{1} \cup X_{2}, X_{1}\right)$ is regular.

Given a real-valued function $f: X \rightarrow R$, we will let $\stackrel{\circ}{X}_{a}^{b}=f^{-1}[a, b)$ and $X_{a}^{b}=f^{-1}[a, b)$.

LEMMA 2. Let $f: X \rightarrow R$ be a submersion (i.e., a differentiable function without critical points), and $(a, b)$ a nonempty interval in $\mathbf{R}$. Then $\left(X_{a}^{b}, \stackrel{\circ}{X_{a}^{b}}\right)$ is regular.

This result depends vitally on a certain "tubular neighborhood property" (see [4, pp. 219-220]) of submersions, which can be described as follows: given a compact subset $C \subset X$ and a point $t \in f(X)$, there exists a closed neighborhood $D$ of $t$ in $f(X)$, a compact subset $F \subset f_{t}$ (where $f_{t}$ denotes the fiber over $t$ ), a subspace $N \subset X$ and a homeomorphism $\phi: D \times F \rightarrow N$, such that

(i) $f(\phi(s, x))=s$ for all $(s, x) \in D \times F$;

(ii) $\phi(s, x)=x$ for all $(s, x) \in D \times F$;

(iii) $C \cap f^{-1}(D) \subset N$.

We will say that $N$ is a tubular neighborhood over $D$ which cuts through $C$. Now let $i_{p}: H_{p}\left(X_{a}^{b}\right) \rightarrow H_{p}\left(X_{a}^{b}\right)$ denote the inclusioninduced homorphism. To prove that $i_{p}$ is injective, let $z$ be a singular p-cycle in $\stackrel{\circ}{X}_{a}^{b}$ and $c$ a singular $(p+1)$-chain in $X_{a}^{b}$ such that $z=\partial c$. By the aforementioned tubular neighborhood property, there exists a tubular neighborhood $N$ over $D=[b-\varepsilon, b]$ which cuts through $|c|$ (the support of $c$ ). Moreover, we may assume that $f^{-1}(D)$ does not meet $|z|$. Now let $Y=X_{a}^{b-\varepsilon} \cap N$, and let $\Pi: Y \rightarrow X_{a}^{b-\varepsilon}$ be the retraction defined by the tubular neighborhood structure of $N$. Then one has (in 
the usual notation)

$$
z=\Pi_{\#}(z)=\partial \Pi_{\#}(c),
$$

which shows that $z$ bounds in $X_{a}^{b-\varepsilon}$.

To prove that $i_{p}$ is surjective, let $z$ be a singular p-cycle in $X_{a}^{b}$ and $N$ a tubular neighborhood over $D=[b-\varepsilon, b]$ which cuts through $|z|$. Let $Y=X_{a}^{b-\varepsilon} \cup N$, and let $\Pi: Y \rightarrow X_{a}^{b-\varepsilon}$ denote again the corresponding retraction. Then $\Pi_{\#}(z)$ is a cycle in $X_{a}^{b-\varepsilon}$, and it remains to show that $\Pi_{\#}(a)$ is homologous to $z$ in $Y$. But this follows from the fact that the tubular neighborhood structure on $N$ enables us to define a (strong) deformation retraction $H: Y \times I \rightarrow Y$ such that $H(x, 1)=\Pi(x)$ for all $x \in Y$.

Our next result will involve the notion of "regular fibers", which we define as follows: a fiber $f_{c}$ is regular if there exists a positive $\varepsilon$ such that the pair $\left(X_{c}^{c+\delta} . f_{c}\right)$ is regular for all $\delta \leq \varepsilon$. Moreover, a point $c \in R$ will be called a regular value of $f$ if $f_{c}$ is a regular fiber.

LEMMA 3. Let $f: X \rightarrow \mathbf{R}$ be a submersion and $(a, b)$ an interval such that every $c \in[a, b)$ is a regular value of $f$. Then $\left(X_{a}^{b}, f_{a}\right)$ is regular.

To prove this, we let $S$ denote the set of all $s \in[a, b]$ such that $\left(X_{a}^{t}, f_{a}\right)$ is regular for all $t \in[a, s]$. Then $S$ must be a subinterval of $[a, b]$ containing the point $a$. Let $c$ denote the least upper bound of $S$. Then $\left(X_{a}^{t}, f_{a}\right)$ is regular for all $t \in[a, c)$. But since singular chains have compact support, this implies that $\left(\stackrel{\circ}{X}_{a}^{c}, f_{a}\right)$ is regular, too. By Lemma 2 this implies that $\left(X_{a}^{c}, f_{a}\right)$ is regular, and hence that $S=[a, c]$.

Now suppose that $c<b$. Since $c$ is a regular value of $f$, there exists a positive $\varepsilon$ such that $c+\varepsilon \leq b$ and $\left(X_{c}^{c+\delta}, f_{c}\right)$ is regular for all $\delta \leq \varepsilon$. Let us choose a positive $\delta \leq \varepsilon$. It now follows from the tubular neighborhood property of a submersion that $\left\{X_{a}^{c}, X^{c+\delta}\right\}$ is an excisive couple (see $\left[4\right.$, p. 222]). Moreover, $\left(X_{c}^{c+\delta}, X_{c}^{c+\delta} \cap X_{a}^{c}\right\}$ is regular, which implies by Lemma 1 that $\left(X_{a}^{c+\delta}, X_{a}^{c}\right)$ is regular, too. But since $\left(X_{a}^{c}, f_{a}\right)$ is regular, one concludes that $\left(X_{a}^{c+\delta}, f_{a}\right)$ is regular. Finally, since this holds for all $\delta \leq \varepsilon, c$ is the least upper bound of $S$. Hence $S=[a, b]$, which implies, in particular, that $\left(X_{a}^{b}, f_{a}\right)$ is regular.

LEMMA 4. Let $f: X \rightarrow \mathbf{R}$ be a submersion and $(a, b)$ an interval such 
that every $c \in[a, b)$ is a regular value of $f$. Then $\left(X^{b}, X^{a}\right)$ is regular (where $\left.X^{t}=f^{-1}(-\infty, t]\right)$.

To prove this, we consider the excisive couple $\left\{X^{a}, X_{a}^{b}\right\}$. By Lemma 3 we conclude that $\left(X_{a}^{b}, X_{a}^{b} \cap X^{a}\right)$ is regular, which implies by Lemma 1 that $\left(X^{b}, X^{a}\right)$ is also regular.

Lemma 5. Let $f: X \rightarrow \mathbf{R}$ be a submersion and $(a, b)$ an interval consisting only of regular values of $f$. For every $t \in(a, b]$, let $i_{p}^{t}$ : $H_{p}\left(f_{a}\right) \rightarrow H_{p}\left(X X_{a}^{t}\right)$ denote the inclusion-induced homomorphism. Then the rank of the kernel and cokernel of $i_{p}^{t}$ are independent of $t$.

Given $a<s<t \leq b$, one has $i_{p}^{p}=j_{p} \circ i_{p}^{s}$, where $j_{p}$ is the inclusioninduced homomorphism $H_{p}\left(X_{a}^{s}\right) \rightarrow H_{p}\left(X_{a}^{t}\right)$. It will suffice to show that $j_{p}$ is an isomorphism. Now, since $\left\{\begin{array}{c}s \\ a\end{array}, X_{s}^{t}\right\}$ is excisive and $\left(X_{s}^{t}, f_{s}\right\}$ is regular by Lemma 3 , the conclusion follows by Lemma 1 .

2. The Morse inequalities. A fiber $f_{c}$ will be called critical if it is not regular. It is critical, therefore, if, for every positive $\varepsilon$, there exists a positive $\delta \leq \varepsilon$ such that $\left(X_{c}^{c+\delta}, f_{c}\right)$ is not regular. If $f_{c}$ is critical, moreover, then $c$ is called a critical value of $f$.

We shall be concerned with submersions $f: X \rightarrow \mathbf{R}$ satisfying three conditions: firstly, $f$ has only a finite number of critical points; secondly, the homology groups $H_{*}\left(X_{a}^{b}\right)$ are all finitely generated; and lastly, $f$ is bounded. The first two conditions suffice to guarantee that all parameters entering into the Morse inequalities are finite, and the last is made for the sake of simplicity (it obviates the appearance of boundary terms). When all these conditions are satisfied we will say that $f$ is of finite type.

Let $f: X \rightarrow \mathbf{R}$ be of finite type and $f_{c}$ a critical fiber. For every $\delta$ and integer $p$ there is an inclusion-induced homomorphism $i_{p}: H_{p}\left(f_{c}\right) \rightarrow H_{p}\left(X_{c}^{c+\delta}\right)$, and Lemma 5 guarantees that the rank of the kernel and cokernel of $i_{p}$ is independent of $\delta$ for $\delta$ sufficiently small (to be precise, $\delta$ must not exceed the distance from $c$ to the next critical 
value of $f$ ). We can therefore define parameters

$$
\begin{array}{r}
d_{p}^{+}(c)=r\left(\operatorname{coker} i_{p}\right) \\
e_{p}^{+}(c)=r\left(\operatorname{ker} i_{p}\right)
\end{array}
$$

where $r$ indicates rank. We may think of $d_{p}^{+}$and $e_{p}^{+}$as the pdimensional "defect" and "excess", respectively, of the given critical fiber. The superscript + , which is superfluous, is there to remind us that we are injecting $f_{c}$ "to the right."

One more remark is in order: the quantities $d_{p}^{+}$and $e_{p}^{+}$could have been defined for regular values of $f$ in just the same way and would turn out to be zero. The vanishing of these parameters is in fact characteristic of regular values.

We now define the "type numbers" $m_{p}^{+}(c)$ of critical fiber by the formula

$$
m_{p}^{+}=d_{p}^{+}+e_{p-1}^{+}
$$

and we let

$$
M_{p}^{+}=\sum_{c} m_{p}^{+}(c)
$$

We can now state our main result:

ThEOREM A. Let $X$ be an $n$-dimensional differentiable manifold and $f: X \rightarrow \mathbf{R}$ a submersion of finite type. Then

$$
\begin{aligned}
M_{0}^{+} & \geq R_{0} \\
M_{1}^{+}-M_{0}^{+} & \geq R_{1}-R_{0} \\
M_{n-1}^{+}-M_{n-2}^{+}+\cdots & \pm M_{0}^{+}=R_{n-1}-R_{n-2}+\cdots \pm R_{0} .
\end{aligned}
$$

where $R_{p}$ denotes the p-dimensional Betti number of $X$.

The proof hinges upon the following results.

LEMMAS 6. If the interval $(a, b)$ contains precisely one critical value $c$, then

$$
m_{p}^{+}(c)=r H_{p}\left(X^{b}, X^{a}\right)
$$


To prove this, we note in the first place that $H_{*}\left(X^{c}, X^{a}\right)$ is zero by Lemma 4 . By the homology sequence of the triple $\left(X^{b}, X^{c}, X^{a}\right)$ we conclude that $H_{*}\left(X^{b}, X^{a}\right) \approx H_{*}\left(X^{b}, X^{c}\right)$. But since $\left\{X^{c}, X_{c}^{b}\right\}$ is excisive, one has $H_{*}\left(X^{b}, X^{c}\right) \approx H_{*}\left(X_{c}^{b}, f_{c}\right)$. Hence it suffices to show that

$$
r H_{p}\left(X_{c}^{b}, f_{c}\right)=d_{p}^{+}+e_{p-1}^{+} .
$$

To this end, we consider the following segment from the exact sequence of the pair $\left(X_{c}^{b}, f_{c}\right)$ :

$$
H_{p}\left(f_{c}\right) \stackrel{i_{p}}{\rightarrow} H_{p}\left(X_{c}^{b}\right) \stackrel{j}{\rightarrow} H_{p}\left(X_{c}^{b}, f_{c}\right) \stackrel{\partial}{\rightarrow} H_{p-1}\left(f_{c}\right) \stackrel{i_{p-1}}{\rightarrow} H_{p-1}\left(X_{c}^{b}\right) .
$$

Since all these groups have finite rank, one now obtains

$$
\begin{aligned}
r H_{p}\left(X_{c}^{b}, f_{c}\right) & =r(\operatorname{ker} \partial)+r(\operatorname{im} \partial) \\
& =r(\operatorname{im} j)+r\left(\operatorname{ker} i_{p-1}\right) \\
& =r H_{p}\left(X_{c}^{b}\right)-r(\operatorname{ker} j)+e_{p-1}^{+} \\
& =r H_{p}\left(X_{c}^{b}\right)-r\left(\operatorname{im} i_{p}\right)+e_{p-1}^{+} \\
& =d_{p}^{+}+e_{p-1}^{+}
\end{aligned}
$$

as was to be shown.

From this point on the proof of Theorem A is standard. We choose a partition $a_{0}<a_{1} \cdots<a_{m}$ such that $f(X) \subset\left(a_{0}, a_{m}\right)$, and each subinterval $\left(a_{i-1}, a_{i}\right)$ contains exactly one critical value. (One might note at this point that $f$ has at least one critical value, inasmuch as $d_{0}^{+}(c)>0$ which $c$ equals the greatest lower bound of $f(x)$ in $\left.\mathbf{R}\right)$.

We now consider the classical equations (first applied to Morse theory in $[\mathbf{1}])$

$$
\begin{aligned}
r H_{p}\left(X^{a_{i}}, X^{a_{i}-1}\right)= & r H_{p}\left(X^{a_{i}}\right)-r H_{p}\left(X^{a_{i-1}}\right) \\
& +S_{p}\left(X^{a_{i-1}}, X^{a_{i}}\right)+S_{p-1}\left(X^{a_{i-1}}, X^{a_{i}}\right),
\end{aligned}
$$

where $S_{q}(A, B)$ denotes the rank of the kernel of the inclusion-induced homomorphism $(H)_{q}(A) \rightarrow H_{q}(B)$. Summing (2.7) over $i$ and applying Lemma 6 gives

$$
M_{p}^{+}=R_{p}+\sum_{i=1}^{m} S_{p}\left(X^{a_{i-1}}, X^{a_{i}}\right)+\sum_{i=1}^{m} S_{p-1}\left(X^{a_{i-1}}, X^{a_{j}}\right),
$$


and taking alternating sums of (2.8) gives

(2.9)

$$
M_{p}^{+}-M_{p-1}^{+}+\cdots \pm M_{0}=R_{p}-R_{p-1}+\cdots \pm R_{0}+\sum_{i=1}^{m} S_{p}\left(X^{a_{i-1}}, X^{a_{j}}\right)
$$

for $p=0,1, \ldots, n-1$. Now, for $p<n-1,(2.9)$ yields the inequalities in (2.4). To complete the proof of Theorem $\mathrm{A}$, one must show that the quantities $S_{n-1}\left(X^{a_{i-1}}, X^{a_{i}}\right)$ are zero. This will be done in the next section.

3. Additional estimates. The following result will yield additional inequalities:

LEMMA 7. If $(a, b)$ contains precisely one critical value $c$, then $S_{p}\left(X^{a}, X^{b}\right) \leq e_{p}^{+}(c)$.

We note, in the first place, that since $\left\{X^{c}, X_{c}^{b}\right\}$ is excisive, one has inclusion-induced isomorphisms

$$
\alpha_{p}: H_{p}\left(X_{c}^{b}, f_{c}\right) \approx H_{p}\left(X^{b}, X^{c}\right)
$$

Moreover, considering the homology sequence of the triple $\left(X^{b}, X^{c}, X^{a}\right)$ and observing that $H_{*}\left(X^{c}, X^{a}\right)$ vanishes by Lemma 4 , one sees that there is an inclusion-induced isomorphism

$$
\beta_{p}: H_{p}\left(X^{b}, X^{a}\right) \approx H_{p}\left(X^{b}, X^{c}\right)
$$

One thus obtains a commutative diagram

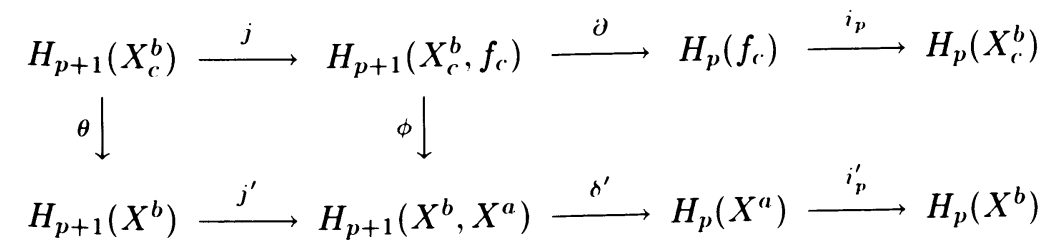

where $\theta$ is inclusion-induced,and $\phi=\left(\beta_{p+1}\right)^{-1} \circ \alpha_{p+1}$. We now define a homomorphism $\psi: \operatorname{ker}\left(i_{p}\right) \rightarrow \operatorname{ker}\left(i_{p}^{\prime}\right)$ by setting $\psi(x)=\delta^{\prime} \circ \phi(y)$, where $y \in \partial^{-1}(x)$. To show that $\psi$ is well-defined, we consider another 
$y^{\prime} \in \partial^{-1}(x)$. Then $\left(y-y^{\prime}\right)$ belongs to the kernel of $\partial$, which is the image of $j$. Now let $z \in H_{p}\left(X_{c}^{b}\right)$ such that $j(z)=y-y^{\prime}$. Then one has

$$
\delta^{\prime} \circ \phi\left(y-y^{\prime}\right)=\delta^{\prime} \circ j^{\prime} \circ \theta(z)=0,
$$

showing that $\delta^{\prime} \circ \phi(y)=\delta^{\prime} \circ \phi\left(y^{\prime}\right)$. Hence $\psi$ is well-defined. Moreover, since $\phi$ is surjective and the kernel of $i_{p+1}^{\prime}$ is the image of $\delta^{\prime}$, it follows that $\psi$, too, is surjective. Hence

$$
S_{p}\left(X^{a}, X^{b}\right)=r\left(\operatorname{ker} i_{p+1}^{\prime}\right) \leq r\left(\operatorname{ker} i_{p+1}\right)=e_{p}^{+}
$$

as was to be shown.

Getting back to (2.9), one now has

$$
\sum_{i=1}^{m} S_{p}\left(X^{a_{i-1}}, X^{a_{i}}\right) \leq E_{p}^{+},
$$

where $E_{p}^{+}=\sum_{c} e_{p}^{+}(c)$. And this proves

THEOREM B. Let $X$ be an n-dimensional differentiable manifold and $f: X \rightarrow \mathbf{R}$ a submersion of finite type. Then

$$
\begin{gathered}
M_{P}^{+}-M_{p-1}^{+}+\cdots \pm M_{0}^{+} \leq R_{p}^{+}-R_{p-1}^{+}+\cdots \pm R_{0}+E_{p}^{+} \\
\text {for } p=0,1, \ldots, n-1 .
\end{gathered}
$$

Now let us observe that no compact fiber of $f$ can be critical (this follows from the tubular neighborhood property). A critical fiber, therefore, is a noncompact manifold of dimension $(n-1)$. Its $(n-1)$ - dimensional homology is consequently zero, which implies that $E_{n-1}^{+}$ vanished. This observation, together with (3.2), completes the proof of Theorem A. $\square$

4. Comparison with the classical case. Let $M$ denote a compact differentiable n-manifold and $h: M \rightarrow \mathbf{R}$ be a real-valued differentiable function with nondegenerate critical points. Let $X$ denote the submanifold of $M$ consisting of all regular points of $h$, and let $f: X \rightarrow \mathbf{R}$ denote the restriction of $h$. Now if $t$ is a regular value of $h$, 
the fiber $f_{t}$ will be compact, and therefore regular (this follows again by the tubular neighborhood property). It follows that $f$ has critical fibers only at the critical values of $h$, and that $f$ is a submersion of finite type. For the sake of simplicity, we shall assume that $h$ has only one critical point corresponding to each critical value.

THEOREM C. Let $c$ be a critical value of $h$ corresponding to a critical point of index $\lambda$. Then

$$
\begin{aligned}
m_{p}^{+}(c)= & \begin{cases}1 & p=\lambda \\
0 & \text { otherwise }\end{cases} \\
& \text { for } p<n-1\left(\text { where } m_{p}^{+} \text {is defined as in }(2.2)\right) .
\end{aligned}
$$

We will need the following elementary result.

LEMMA 8. The inclusion $X^{t} \subset M^{t}$ induces isomorphisms $H_{p}\left(X^{t}\right) \approx$ $H_{p}\left(M^{t}\right)$ for every regular value $t$ of $h$ and $p<n-1$.

To prove this, we enclose each critical point of $h$ belonging to $M^{t}$ in a small regularly-imbedded n-ball, and let $I$ denote the union of these balls. Then $\left\{X^{t}, W^{t}\right\}$ is an excisive couple with $X^{t} \cup W^{t}=M^{t}$. Moreover, the intersection $X^{t} \cap W$ has the homology type of $r$ disjoint $(n-1)$-spheres, where $r$ is the number of critical points in $M^{t}$. The desired conclusion follows easily from the Maver-Vietoris sequence of the couple $\left\{X^{t}, W\right\}$.

Now to the proof of Theorem C: Let $(a, b)$ denote an interval containing $c$ as its only critical value, and consider the commutative diagram

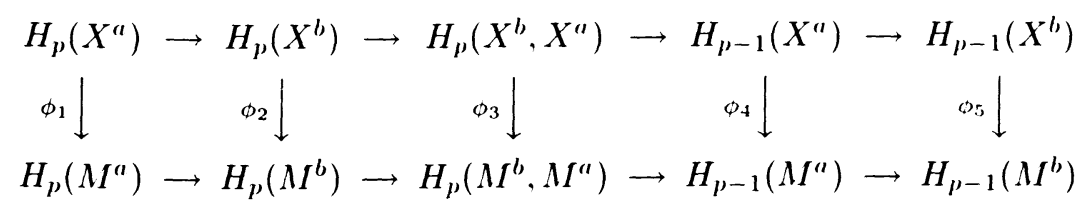


where the homomorphisms $\phi_{i}$ are inclusion-induced, and each row belongs to the homology sequence of the corresponding pair. Lemma 8 and the 5-lemma now imply that $\phi_{3}$ is an isomorphism. This fact, together with Lemma 6 , implies that

$$
m_{p}^{+}(c)=r H_{p}\left(M^{b}, M^{a}\right)
$$

The desired conclusion follows now from a well-known result of classical Morse theory (see p. $29[\mathbf{2}]$ ).

We are now in a position to see that, for $p<n-1$, our inequalities

$$
M_{p}^{+}-M_{p-1}^{+}+\cdots \pm M_{0}^{+} \geq R_{p}-R_{p-1}+\cdots \pm R_{0}
$$

associated with the submersion $f$ do in fact reduce to the classical Morse inequalities for $h$. For, by Theorem C, we conclude that $M_{p}^{+}$is just the number of critical points of $h$ with index $p$, and by Lemma 7 that $R_{p}$ is indeed the p-dimensional Betti number of $M$ when $p<n-1$.

A few general remarks may be in order at this point. Classically, one defines the type of numbers of a critical point $x$ by the formula

$$
m_{p}=r H_{p}\left(M^{c}, \stackrel{\circ}{M^{c}}\right)
$$

where $c=h(x)$ and $\stackrel{\circ}{M}^{c}=h^{-1}(-\infty, c)$. For the corresponding submersion $f=h \mid X$, on the other hand, one finds that $H_{p}\left(X^{c}, \stackrel{\circ}{X}^{c}\right)$ is zero for all $p$ (as we learn from Lemma 2). Again, our "submersion approach" to Morse theory hinges upon the parameters $d_{p}^{+} \& e_{p}^{+}$, which measure the extent to which the inclusion-induces homomorphism $i_{p}: H_{p}\left(f_{c}\right) \rightarrow H_{p}\left(X_{c}^{c+\varepsilon}\right)$ deviates from an isomorphism. But is easy to see that the corresponding maps $i_{p}: H_{p}\left(h_{c}\right) \rightarrow\left(M_{c}^{c+\varepsilon}\right)$ defined with respect to $h$ are always isomorphisms (because $M_{c}^{c+\varepsilon}$ ) can be retracted onto the critical fiber $h_{c}$ along the "gradient lines"). Thus we find that the presence or absence of the critical point has a decisive effect on the homology structure of corresponding "tubular neighborhoods." The distinctive feature of our approach is to consider the structure of these tubular neighborhoods in the absence of critical points. 
5. The classification of critical points. Morse (see [3]) has distinguished two types of critical points, based on the fact that if $h: M \rightarrow \mathbf{R}$ has a single critical point of index $\lambda$ corresponding to the critical value $c$, then $r H_{p}\left(M^{t}\right)$ behaves in one of two ways as $t$ increases from $c-\varepsilon$ to $c+\varepsilon$ : either it increases by 1 in dimension $\lambda$, or else it decreases by 1 in dimension $\lambda-1$ (while in either case it remains unchanged in the other dimensions). Morse speaks thus of critical points of increasing and decreasing type. Now it is of interest to note that the submersion approach leads to a similar dichotomy in the classical case when the index $\lambda$ is less than $n-1$. For if $c$ is a critical value of $h$ corresponding to a single critical point of index $\lambda<n-1$, then one concludes by Theorem $\mathrm{C}$ that

$$
d_{\lambda}^{+}+e_{\lambda-1}^{+}=1 \text {, }
$$

which obviously can happen only in one of two ways (either $d_{\lambda}^{+}=1$ and $e_{\lambda-1}^{+}=0$, or the other way round). Thus, again, critical points are divided into two categories: type $\mathrm{D}$ and type $\mathrm{E}$, let us say. It is a likely conjecture, moreover, that the two classifications coincide, and that, in fact, increasing type corresponds to type $\mathrm{D}$ and decreasing type of type E. But it happens that this conjecture is only half correct.

It is easy to see that a critical point of type $\mathrm{D}$ (and index $\lambda<n-1$ ) is of increasing type. For in that case, $e_{\lambda-1}^{+}$is zero, which implies by Theorem $\mathrm{C}$ that $e_{p}^{+}$is zero for all $p<n-2$. Thus, in particular, $e_{\lambda-1}^{+}$ and $e_{\lambda-2}^{+}$are both zero, which implies by Lemma 7 that $S_{p}\left(X^{c-\varepsilon}, X^{c+\varepsilon}\right)$ vanishes likewise in these dimensions. Hence, by (2.7), one now obtains

$$
r H_{\lambda-1}\left(C^{c+\varepsilon}, X^{c-\varepsilon}\right)=r H_{\lambda-1}\left(X^{c+\varepsilon}\right)-r H_{\lambda}\left(X^{c-\varepsilon}\right) .
$$

But, on the strength of Lemma 8, this implies that

$$
r H_{\lambda-1}\left(M^{c+\varepsilon}\right) \geq r H_{\lambda-1}\left(M^{c-\varepsilon}\right),
$$

which excludes the possibility that the critical point is of decreasing type.

On the other hand, it is not true that a critical point of increasing type need be of type D. We will consider a simple example. Let $T \subset \mathbf{R}^{3}$ denote a torus of outer diameter 3 and inner diameter 1 . We will 
suppose that $T$ is "standing on end" (like a tire rolling on the xy-plane). One sees now that the height function $z: T \rightarrow \mathbf{R}$ has four critical points $p_{i}(i=0,1,2,3)$ with critical values $z\left(p_{i}\right)=i$. Let $M=T \times S^{1}$ (where $S^{1}$ denotes the unit circle), and let $h: M \rightarrow \mathbf{R}$ be defined by the formula

$$
h(u, \theta)=z(u)+2 \cos \theta .
$$

This function has eight critical points, namely, $\left(p_{i}, 0\right)$ and $\left(p_{i}, \pi\right)$. The critical point $\left(p_{0}, 0\right)$, in particular, has index 1 and is easily seen to be of increasing type (this follows from the fact that $M^{c-\varepsilon}$ is obviously connected, and $M^{c+\varepsilon}$ has the homotopy type of $M^{c-\varepsilon}$ with a 1-cell attached). But one can also see that $\left(p_{0}, 0\right)$ is of type E. Now the fibers of the associated submersion $f: X \rightarrow \mathbf{R}$, being 2-dimensional manifolds, are easy to describe. The critical fiber $f_{c}$ consists in fact of two punctured tori, whereas $f_{c+\varepsilon}$ is a double torus. The space $X_{c}^{c+\varepsilon}$ is therefore connected while $f_{c}$ is not, proving that $e_{0}^{+}=1$. The critical point is therefore of type $\mathrm{E}$.

On the strength of these observations we are now in a position to interpret the inequalities (3.2) in the classical case (where $f=h \mid X$ ). In the light of what has been said concerning the classification of critical points, we see that, for $p<n-2$, the parameter $E_{p}^{+}$constitutes an upper bound for the number $\mu_{p+1}^{-}$of critical points of index $p+1$ and decreasing type. Thus (3.2) accords with the classical formula (see $[\mathbf{3}$, p. 560]).

$$
M_{p}-M_{p-1}+\cdots \pm M_{0}=R_{p}-R_{p-1}+\cdots \pm_{0}+\mu_{p+1}^{-},
$$

where $M_{q}$ denotes the total number of critical points of index $q$, and $R_{q}$ denotes the $q$-dimensional Betti number of $M$.

\section{REFERENCES}

1. W. Mayer, A new approach to critical point theory, Bull. Amer. Math. Soc. 46 (1940), 838-847.

2. J. Milnor, Morse Theory, Princeton University Press, 1963.

3. M. Morse and G.B. Van Schaak, The critical point theory under general boundary conditions, Ann. Math. 35 (1934), 545-571. 
4. J.W. Smith, On the homology structure of submersions, Math. Ann. 193 (1971), 217-224.

5. E.H. Spanier, Algebraic Topology, McGraw Hill, 1966.

Department of Mathematics. Oregon State University. Corvallis. OR 97331-4605 
